\title{
A de novo missense mutation in a critical domain of the X-linked DDP gene causes the typical deafness-dystonia-optic atrophy syndrome
}

\author{
Lisbeth Tranebjærg ${ }^{1}$, Ben CJ Hamel ${ }^{2}$, Fons JM Gabreels ${ }^{3}$, Willy O Renier ${ }^{3}$ and \\ Marijke Van Ghelue
}

${ }^{1}$ Department of Medical Genetics, University Hospital of Tromsø, Norway; ${ }^{2}$ Department of Human Genetics,
Division of Clinical Genetics; ${ }^{3}$ Department of Child N eurology, University Hospital Nijmegen, The Netherlands

We report the first de novo mutation in the DDP gene in a Dutch 11-year-old boy with deafness and dystonia. Previously reported mutations in the DDP gene have all been frameshifts/nonsense mutations or deletion of the entire gene as part of a larger deletion encompassing the BTK gene. The clinical presentation was uniformly characterised by sensorineural hearing loss, dystonia, mental deterioration, paranoid psychotic features, and optic atrophy, indicating progressive neurodegeneration. Our report illustrates that de novo mutations occur and that a missense mutation, $\mathrm{C66 \textrm {W }}$, may cause an equally severe clinical picture. The diagnosis of sensorineural hearing impairment associated with neurologic and visual disability in a male, therefore, should encourage the search for mutations in the DDP gene, even in sporadic cases. The association of deafness-dystonia syndrome with a missense mutation provides valuable information for in vitro investigations of the functional properties of the deafness-dystonia peptide which was recently shown to be the human homolog of a yeast protein, Tim8p, belonging to a family of small Tim proteins involved in intermembrane protein transport in mitochondria. European Journal of Human Genetics (2000) 8, 464-467.

Keywords: DDP gene; mitochondrial dysfunction; dystonia; hearing impairment

\section{Introduction}

Mohr-Tranebjærg syndrome (MIM 304700) is characterised by $\mathrm{X}$-linked recessive progressive sensorineural hearing loss, dystonia, dementia, psychotic features and optic atrophy and the disease gene was mapped to Xq22. ${ }^{1-3}$ In 1996, the DDP gene was characterised and disease-causing mutations were reported in the original Norwegian and two other families. ${ }^{4}$ The DDP CDNA sequence is $1167 \mathrm{bp}$ in length excluding the poly $(A)^{+}$tail. ${ }^{4}$ The open reading frame encodes a 97 aminoacid polypeptide. The DDP gene contains two exons and a single intron of approximately $2 \mathrm{~kb}^{4}$ Strong homology to a predicted polypeptide (SPAC13G6.04) from an S.Pombe gene was found but the function in yeast was unknown at that

Correspondence: Lisbeth Tranebjærg, PhD, MD, Department of Medical Genetics, University Hospital of Tromsø, N-9038 Tromsø, Norway. Fax: +47 77645430; Tel: +47 77645410; E-mail: lisbetht@fagmed.uit.no Received 20 October 1999; revised 14 February 2000; accepted 24 February 2000 time. $^{4}$ Subsequently, Jensen syndrome (MIM 311150) was reported to be an allelic disorder caused by a nonsense mutation in the same gene, ${ }^{5-7}$ (unpublished data). Different mutations in the DDP gene were identified in three families from Australia and the Netherlands ${ }^{8}$ (unpublished data), and the clinical presentation was somewhat broadened because the hearing impairment was either congenital or onset in childhood and the neurological abnormality was either ataxia or dystonia. Progressive neurodegeneration was consistently found but time of onset and rate of progression of the disease have been more variable than was first described in the original Norwegian family. ${ }^{1,2}$ All mutations so far have been predicted to cause absent or truncated gene product. Recent characterisation of a likely mitochondrial transport function of the DDP polypeptide ${ }^{9,10}$ makes the identification of a missense mutation extremely valuable for further investigation of the functional properties of the protein. 


\section{Materials and methods \\ Family material}

A Dutch male patient with a negative family history had hearing impairment and dystonia resembling Mohr-Tranebjærg syndrome.

\section{DNA sequencing and microsatellite analysis}

DNA was extracted from peripheral leucocytes and cultivated fibroblasts in accordance with routine procedures and sequencing was done according to previously reported methods. ${ }^{4}$ The coding region spanning both exons was amplified by PCR using the primers as described. ${ }^{4}$ Both strands of the PCR product were sequenced using the dideoxy termination method provided by the manufacturer (sequenase PCR product list, USB). Detection of the $233 \mathrm{C} \rightarrow \mathrm{G}$ substitution in 48 normal controls was performed by amplying exon 2 with sense primer 5'-TTATTCTGTATCTTGTCCTGAGCAG-3' (DDP3-14) ${ }^{4}$ and anti-sense primer 5'-GCTGTITCTTCAATCATCCCAGTAACAGC-3' (DDP PBmrl pr). The nucleotides underlined were modified in order to introduce an internal $\mathrm{Bmrl}$ restriction site within the primer to ensure that the PCR products were fully digested. The PCR products were $264 \mathrm{bp}$ (undigested product), $244 \mathrm{bp}$ $+20 b p$ (digested normal), $244 b p+155 b p+89 b p+$ $20 \mathrm{bp}$ (digested carrier 233G), and $155 \mathrm{bp}+89 \mathrm{bp}+20 \mathrm{bp}$ (digested hemizygous 233G).

The X-linked microsatellite markers DXS6803 (Xq21.3) and DXS6804 (Xq23) spanning a genetic region of approximately $25 \mathrm{cM}$ containing the DDP gene were typed in order to determine the origin of the mutated $X$ chromosome in the patient's maternal grandparents.

\section{Results}

\section{Case report}

A Dutch 11-year-old male from a family with a negative family history was diagnosed with sensorineural hearing impairment at age 21/2 years, mild mental retardation (no formal testing), and normal behaviour. He was born at home at expected time without birth-related complications. His BW was $3600 \mathrm{gs}, \mathrm{BL} 51 \mathrm{~cm}$, and Apgar score normal. He walked without support at age 14 months, and said his first words at age 14 months but further language development stagnated from age 18 months. He spoke in sentences at age 44 months. He was toilet-trained during daytime by age 4 . At the age of 3 years a neurol ogical examination was normal but at age 5-6years he had lost skills such as drawing, playing with puppets, and similar tasks. From age 10, he had abnormal posture and trembling of the left hand, and increasing difficulties with tieing his shoelaces and his belt. Several minor traumata occurred because of frequent stumbling and falling. He had slight hyperflexia, dyspraxia, poor synergia and many synkinesias and progressive dystonia, especially of the left upper extremity, as well as atrophy of the small muscles of the left hand. Cerebral CT and MRI scans remained normal. At age 11 1/12 years his height, weight and OFC were all within -1SD. Light microscopic examination of a needle muscle biopsy was unremarkable and EMG was myopathic. EM analysis revealed accumulation of abnormal amounts of fatty vacuoles but no gross abnormalities of the mitochondria. Biochemical analysis of muscle tissue from an open biopsy are pending. He had no subjective visual complaints but a Visual Evoked Potential (VEP) examination done after the identification of a DDP mutation showed prolonged latency times in accordance with incipient optic atrophy. After demonstration of a base substitution, subsequent sequencing was performed on DNA from his mother, maternal grandparents, and other relevant family members as illustrated in Figure1.

\section{Mutation detection}

After sequencing both exons of the DDP gene a transversion from $C \rightarrow G$ at nucleotide position 233 was detected in the patient, IV:6, and his mother, III:6 (Figures 1 and 2) leading to a change from TGC $\rightarrow$ TGG at codon 66 . This mutation removes a cysteine and introduces a tryptophan, C66W. No mutation was detected in the maternal grandparents or the maternal sister. In addition, DNA extracted from cultivated fibroblasts of 11:4 was sequenced and showed the normal sequence (Figures 1 and 2 ). The mutation was not present in any of the 48 Norwegian control males studied.

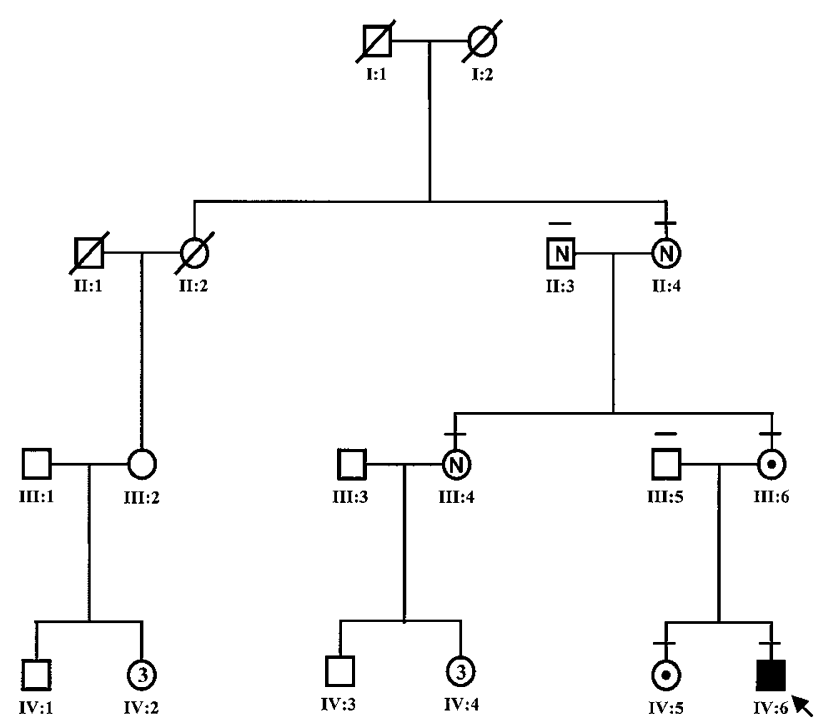

Figure 1 Pedigree of the family. All investigated members are marked with a bar. Proven carriers are indicated by a dot, and $\mathrm{N}$ denotes absence of the mutation. Sequencing was also performed on extracted DNA from cultivated fibroblasts in II-4, in order to detect possible germline mosaicism, which could not be demonstrated. 


\section{$233 \mathrm{C} \longrightarrow \mathrm{G}, \mathrm{C} 66 \mathrm{~W}$}
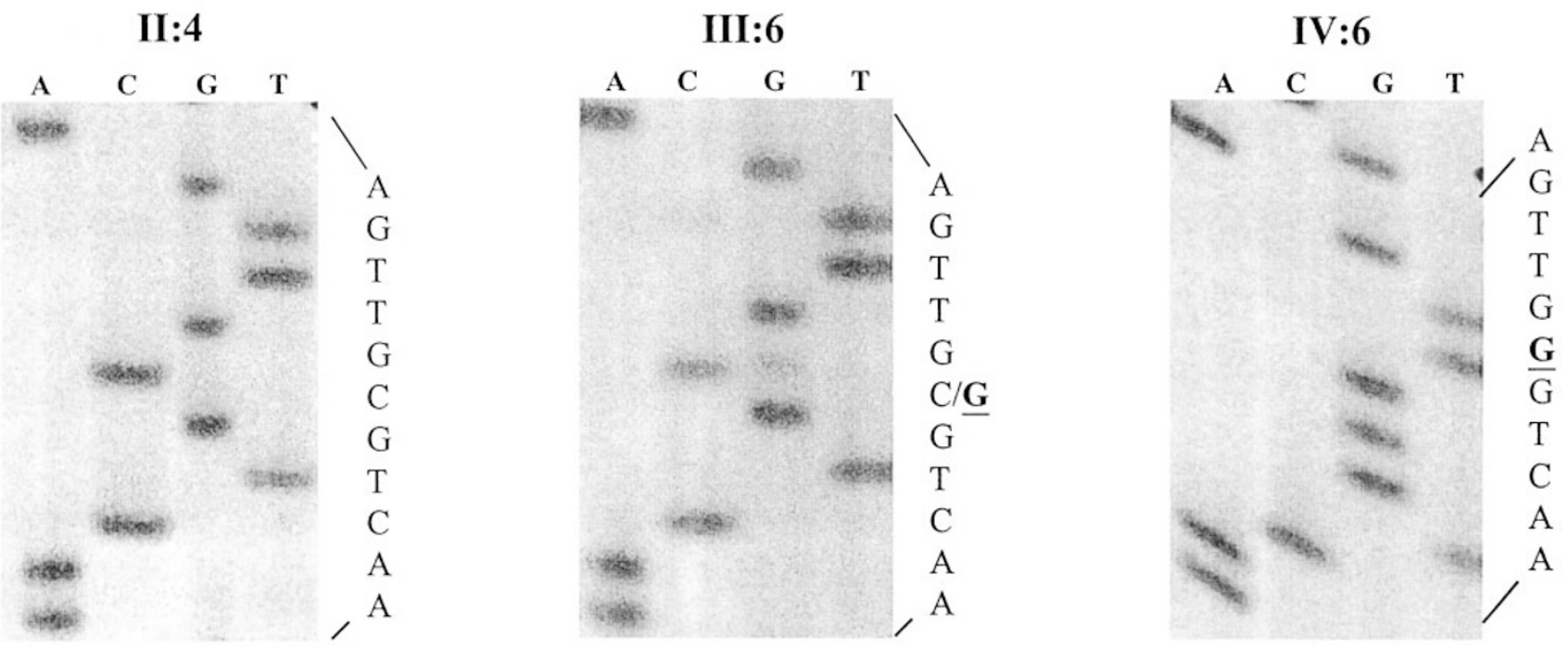

159 CTTTGTTGGGAGAAGTGCATGGACAAGCCTGGGCCAAAGTTG

$\begin{array}{lllllllllllllllll}42 & \mathrm{~L} & C & \mathrm{~W} & \mathrm{E} & \mathrm{K} & \boldsymbol{C} & \mathrm{M} & \mathrm{D} & \mathrm{K} & \mathrm{P} & \mathrm{G} & \mathrm{P} & \mathrm{K} & \mathrm{L}\end{array}$

GACAGTCGGGCTGAGGCCTGTTTTGTGAACTGCGTTGAGCGC 242

$\begin{array}{lllllllllllllllll}\mathrm{D} & \mathrm{S} & \mathrm{R} & \mathrm{A} & \mathrm{E} & \mathrm{A} & \boldsymbol{C} & \mathrm{E} & \mathrm{V} & \mathrm{N} & \boldsymbol{C} & \mathrm{V} & \mathrm{E} & \mathrm{R} & 69\end{array}$

Figure 2 Partial sequence of the DDP gene and partial amino acid composition of DDP. The sequence change $233 \mathrm{C} \rightarrow \mathrm{G}$ is shown leading to $\mathrm{C} 66 \mathrm{~W}$ codon change in the patient, IV:6, his carrier mother, III:6, and normal sequence in his maternal grandmother, II:4. The lower half shows codons starting from codon 42 , and highlights (in bold) the two regions with a $\mathrm{C}-\mathrm{X}_{3}-\mathrm{C}$ domain at codon $43-47$ and at codon 62-66. The base pairs in bold in the bottom section represent the normal DDP sequence (top left illustration). The Cs in these two $\mathrm{C}-\mathrm{X}_{3}-\mathrm{C}$ domains are completely conserved in all identified yeast and human Tim polypeptides. The present missense mutation disrupts the last $\mathrm{C}$ in the second $\mathrm{C}-\mathrm{X}_{3}-\mathrm{C}$ domain, which is probably deleterious to an important region, either involved in disulfide bridge formation or a $\mathrm{Zn}$ finger binding function.

\section{Discussion}

Deletion of the entire DDP gene or frameshift / nonsense mutations, ${ }^{4}$ (un published data) cause a severe, al beit variable, clinical phenotype as previously described briefly in two families, ${ }^{4}$ and extensively in four subsequently identified unrelated families, ${ }^{4}$ (unpublished data). In 1975, Scribanu and Kennedy ${ }^{11}$ described a deafness-dystonia-optical atrophy disorder very similar to the M ohr-Tranebjærg syndrome but no mutation in the DDP gene in that American family has so far been reported. An Australian family with features suggestive of a DDP mutation has recently been reported, ${ }^{8}$ and a mutation in the DDP gene has been identified (unpublished data). A number of clinically similar disorders with $\mathrm{X}$-linked recessive inheritance have been shown not to be caused by mutations in the DDP gene (MIM 301835, 312910, 301840, 312840, 301790, 311050 (L Tranebjærg et al unpublished data). Only Jensen syndrome (MIM 311150) turned out to be an allelic condition also caused by a nonsense mutation in the DDP gene, (un published data). In total, DDP mutations have been identified in seven families worldwide, ${ }^{4}$ (unpublished data).

The detection of a mutation in a sporadic male patient with deafness and dystonia illustrates several interesting features about the DDP gene. First, it represents the first documented case of a de novo mutation, and second, it represents a different type of mutation from those previously reported. We found normal DNA sequence of the DDP gene in lymphocyte DNA from both grandparents (Figure 1, II:3 
and $1 \mathrm{I}: 4)$. Additional sequencing of extracted DNA from fibroblasts from a peripheral skin biopsy of the maternal grandmother (Figure1, II:4) also showed normal DDP sequence. Subsequent microsatellite typing of the patient, his mother and her parents showed that the $X$ chromosome carrying the mutation in the DDP gene was of grandmaternal origin (data not shown). Our results are compatible with the occurrence of a mutation in the germ cells of the maternal grandmother, but we are unable to determine if she is mosaic for the mutation in the germ cells. The diagnosis of a de novo mutation illustrates the relevance of considering MohrTranebjærg syndrome in clinically suspicious cases, even with sporadic occurrence. The small size of the gene makes the sequencing straightforward in a diagnostic laboratory. Clinically, the present patient cannot be distinguished from published patients with mutations leading to absent or truncated polypeptide, ${ }^{4}$ (unpublished data). We therefore conclude that this strongly indicates that the missense mutation has disrupted a functionally important domain of the DDP protein. The disease-causing role of the present mutation is supported by the absence of the mutation in 48 normal $X$ chromosomes. The published mutations, ${ }^{4}$ (unpublished data), were all located upstream from the missense mutation reported here. This small polypeptide is now presumed to have an intermembrane transport function in mitochondria based on recent yeast research., ${ }^{9,10}$ The identification of a new family of small mitochondrial import proteins, Tim proteins, provides an opportunity to investigate the functional consequences of mutations in the DDP gene. The amino acid change from a cysteine (uncharged, with a sulphur group) to a tryptophan (non-polar) is dramatic and it disrupts the second of two identical domains, $\mathrm{C}-\mathrm{X}_{3}-\mathrm{C}$, consisting of two cysteines separated by three other amino acids and each domain separated by 14 codons (Figure2). This motif is reminiscent of a canonical zinc finger, but differs from it by length of interspacer residues. ${ }^{10}$ Some data support that the small Tim proteins bind zinc and that zinc binding is required for their function in vivo, but convincing evidence has not yet been published. ${ }^{10,12}$ Another possibility is that the cysteines from a disulfide bridge which is disrupted by this mutation. The complete conservation of these four cysteines in the coding sequence of all the small Tim proteins characterised so far, DDP1 (formerly known as DDP), DDP2, hTim 9, hTim 13, strongly suggest that they are part of important functional domains. ${ }^{9,10}$ The experiments performed by Koehler et $\mathrm{al}^{9}$ also showed that human DDP1, like its yeast homolog Tim8p, is located in the mitochondrial intermembrane space, and that Tim8p and Tim13p form a 70-kDa complex involved in essential import pathway in mitochondria.

The absence of hTim 13 protein associated with absent DDP1 in human fibroblast cells from affected male patients from the original family with Mohr-Tranebjærg syndrome, ${ }^{2}$ (unpublished data) strongly suggests that DDP1 is functionally related to and a partner of hTim 13. Future studies of yeast and human Tim proteins are foreseen with great expectations for understanding the pathogenesis of the progressive neurodegeneration secondary to the apparent disruption of the oxidative phosphorylation in mitochondria, and will hopefully lead to some kind of treatment in human patients.

\section{Acknowledgements}

Financial support was provided from the Norwegian Research Council (grant 114575/320) and from the Danish Research Foundation for Studies of Deafness and Hearing Impairment. (Forskningsfondet for studier af døvhed og tunghørhed).

\section{References}

1 Mohr J, Magerøy K: Sex-linked deafness of a possibly new type. Acta Genet Stat Med (Basel) 1960; 10: 54-62.

2 Tranebjærg L, Schwartz C, Eriksen $\mathrm{H}$ et al: A new $\mathrm{X}$ linked recessive deafness syndrome with blindness, dystonia, fractures, and mental deficiency is linked to Xq22. J Med Genet 1995; 32: 257-263.

3 Ponjavic V, Andreasson S, Tranebjærg L, Lubs H: Full-field electroretinograms in a family with Mohr-Tranebjærg Syndrome. Acta Ophthalmol Scand 1996; 74: 632-635.

4 Jin $H$, May M, Tranebjærg L et al: A novel $X$-linked gene, DDP, shows mutations in families with deafness (DFN-1), dystonia, mental deficiency and blindness. Nat Genet 1996; 14: 177-180.

5 Jensen PKA: Nerve deafness, optic nerve atrophy and dementia: A new X-linked recessive syndrome? Am J Med Genet 1981; 9: $55-60$.

6 Jensen PKA, Reske-Nielsen E, Hein-Sørensen O, Warburg M: The syndrome of optico-acoustic nerve atrophy with dementia. Am J Med Genet 1987; 28: 517-518.

7 Reske-Nielsen E, Jensen PKA, Hein-Sørensen O, Abelskov K: Calcification of the central nervous system in a new hereditary neurological syndrome. Acta Neuropathol 1988; 75: 590-596.

8 Hayes MW, Ouvrier R, Evans W, Somerville E, Morris JG: X-linked dystonia-deafness syndrome. Mov Disord 1998; 13: 303-308.

9 Koehler C, Leuenberger D, Merchant S, Renold A, Junne T, Schatz G: Human deafness dystonia syndrome is a mitochondrial disease. Proc Natl Acad Sci USA 1999; 96: 2141-2146.

10 Koehler CM, Merchant S, Schatz G: How membrane proteins travel across the mitochondrial intermembrane space. Trends Biochem Sci 1999; 24(11): 428-432.

11 Scribanu N, Kennedy C: Familial syndrome with dystonia, neural deafness, and possibly intellectual impairment: clinical course and pathological findings. Adv Neurol (Dystonia) 1975; 14: 235-243.

12 Jin H, Kendall E, Freeman TC, Roberts RG, Vetrie DLP: The human family of Deafness/ Dystonia/ Dystonia Peptide (DDP) related mitochondrial import proteins. Genomics 1999; 61: 259-267. 